\title{
Covid-19 Pandemic: A Focused Review on Paediatric Patients
}

\author{
Nurun Nahar Fatema ${ }^{1}$, Nure Ishrat Nazme ${ }^{2}$, Md. Ashfaque Ahemmed Khan ${ }^{2}$, Abdullah Al-Amin ${ }^{2}$, \\ ATM Asadullah Kaisar Sujan² \\ ${ }^{1}$ Dept. of Paediatrics, CMH, Dhaka, Dept. of Paediatrics, AFMC, Dhaka, Dept. of Paediatric \\ Cardiology, CMH \& AFMC, Dhaka, ${ }^{2}$ Dept. of Paediatrics, CMH, Dhaka
}

Abstract:

Keywords:

From last two decades, coronaviruses have led to three major outbreaks that started from 2002 with SARS-CoV-2, SARS-CoV, then MERS-CoV in 2012 and currently SARS-CoV-2 (COVID-19). There is limited data Paediatric. regarding the demographics and clinical features of SARS-CoV-2 infection in children. This information is especially important as pneumonia is the single leading cause of death in children worldwide. This Systematic Review aims to elucidate a better understanding of the epidemiologic, clinical and diagnostic findings, treatment and prevention options as well as global impact of COVID19 on the pediatric population with a focus on status in Bangladesh.

(Cardiovasc. j. 2020; 13(1): 62-74)

\section{Background:}

COVID-19, the disease caused by the novel coronavirus SARS-CoV-2, has led to unprecedented global pandemic affecting persons of all ages. The first case of COVID-19 caused by severe acute respiratory syndrome-coronavirus 2 (SARS-CoV2) was reported in November 2019. ${ }^{1}$ After an outbreak of unusual respiratory disease causing severe cases of pneumonia initially in Wuhan, China, caused by novel enveloped RNA coronavirus, the World Health Organization (WHO) named the virus, the 2019 novel coronavirus (2019-nCoV) on January 7, 2020. ${ }^{2}$ Soon after, the virus rapidly spread throughout the world. On February 11, 2020, WHO named the illness associated with $2019-\mathrm{nCoV}$ the 2019 novel coronavirus disease (COVID-19). ${ }^{3}$

The rapid progression to a global pandemic of COVID-19 has had profound medical, social, and economic consequences. Children represent a vulnerable population in any type of pandemic. The clinical characteristics, disease progression and outcome in children so far appeared milder in majority (>90\%) of paediatric patients compared to older individuals although the scenario are evolving. Paediatric patients with COVID-19 are mostly owing to family cluster or with a close contact history. However, the precise impact of this novel virus on paediatric population remains uncertain. ${ }^{4}$

\section{SARS CoV-2: Origin and epidemiology \\ Origin-}

An outbreak of unusual respiratory disease causing severe cases of pneumonia initially in Wuhan, Hubei Province, China was caused by a novel enveloped RNA coronavirus on 31 December 2019. Since late February, the majority of cases reported are from outside China, with an increasing majority of these reported from EU/ EEA countries and the UK. World health organization (WHO) declared the out break a " Public health emergency of international concern "on $30^{\text {th }}$ January 2020 and a "Pandemic" on $11^{\text {th }}$ March 2020. ${ }^{5}$

\section{Epidemiology-}

From the beginning of this outbreak, the percentage of children within the total COVID-19 patients was small. According to the data of the China CDC from February 2020, children younger

Address of Correspondence: Brig. Gen. Nurun Nahar Fatema. Dept. of Paediatrics, CMH, Dhaka, Dept. of Paediatrics, AFMC, Dhaka, Dept. of Paediatric Cardiology, CMH \& AFMC, Dhaka, Bangladesh. Email colfatema@hotmail.com

- 2020 authors; licensed and published by International Society of Cardiovascular Ultrasound, Bangladesh Chapter and Bangladesh Society of Geriatric Cardiology. This is an Open Access article distributed under the terms of the CC BY NC 4.0 (https://creativecommons.org/licenses/by-nc/4.0) 
than 10 / years of age and within the age of 11-19/ years occupied $1 \%$ each of the total cases. Considering this age group represents $20 \%$ of the total population, this may indicate less prevalence of COVID-19 in pediatric population. However, this may be underestimation of actual incidence in pediatric population if less tests were undertaken in children due to less symptoms. One confounding factor is that schools in China were closed for most of the epidemic due to the Chinese New Year holidays, which might have contributed to less exposure among children. ${ }^{6}$

On April 6, 2020, the US CDC released the study of 2572 COVID-19 cases among children younger than 18/ years. Of all reported cases in the US, this occupied only $1.7 \%$ of the total cases. Overall, the data suggested that children were less symptomatic than adults as in Chinese reports. ${ }^{5}$ Until 16 August 2020, the number of confirmed cases worldwide was recorded to be 21294845 with 761779 deaths deaths reported to WHO. Most of the reported cases were adults with few cases described in children and neonates. ${ }^{7}$

\section{SARS CoV-2: Classification and transmission}

Classification-

SARS-CoV-2 belongs to the Sarbecovirus subgenus of the Coronaviridae family, and is the seventh coronavirus known to infect humans. The virus has been found to be similar to SARS-like coronaviruses from bats, but it is distinct from SARS-CoV and MERS-CoV. Different types of human coronaviruses vary in how severe the resulting disease becomes, and how far they can spread. Doctors currently recognize seven types of coronavirus that can infect humans. ${ }^{8}$

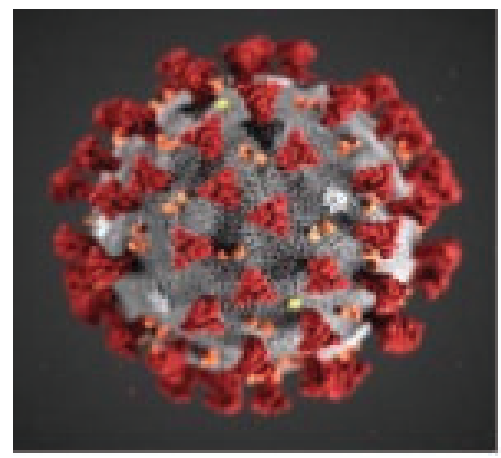

Fig.-1: Picture showing ultrastructural morphology of severe acute respiratory syndrome coronavirus 2 (SARS-CoV-2) when viewed with electron microscopically (Centers for Disease Control and Prevention) ${ }^{9}$
SARS-CoV-2 belongs to Betacoronavirus together with two highly pathogenic viruses, SARS-CoV and MERS-CoV. SARS-CoV-2 is an enveloped and positive-sense single-stranded RNA (+ssRNA) virus. SARS-CoV-2 is considered a novel human-infecting Betacoronavirus. ${ }^{9}$

\section{Transmission-}

Limited research is available on how human corona virus $(\mathrm{HCoV})$ spreads from one person to the next. However, According to current evidence, SARS-CoV-2, the virus that causes COVID-19, is primarily transmitted between people through respiratory droplets and contact routes. ${ }^{10}$ The initial infections were linked to Huanan Seafood market in China, mostly due to animal contact. However, COVID-19 is not considered as direct zoonosis as its transmission now is primarily human to human. ${ }^{11}$

The mode of transmission of the virus between humans is via respiratory droplets. ${ }^{12}$ Transmission may also occur through fomites in the immediate environment around the infected person. Therefore, transmission of the COVID-19 virus may occur by direct contact with infected people and indirect contact with surfaces in the immediate environment or with objects used on the infected person (e.g. stethoscope or thermometer). ${ }^{13}$

Vertical transmission is still a matter of debate and yet to be established. In two studies performed on pregnant women with confirmed COVID-19 infection who delivered by either normal vaginal delivery or caesarian section, all neonates tested negative for COVID-19. ${ }^{14,15}$ Another study has found that 3 out of 33 neonates born to COVID-19 positive mothers had positive nasopharyngeal and rectal swabs for COVID-19. ${ }^{16}$

Children appear less likely than adults to be the first cases of COVID-19 within a household, based on data from families of 39 children younger than 16 years. ${ }^{17}$ These findings support data from previous studies suggesting that children mainly become infected from adult family members rather than transmitting the virus to them, the researchers said.

\section{Pathogenesis and immune response of COVID-19: ${ }^{18,19}$}

The current working hypothesis for the pathogenesis of COVID-19 suggests that the highly- 
expressed angiotensin-converting enzyme 2 (ACE2) in the airway epithelia and particularly the alveoli acts as primary gateway for the cellular penetration of SARS-CoV-2 causing inflammation and local cell death as SARS-CoV-2 is primarily a lung pathogen.

\section{Case definitions for surveillance:}

Case and contact definitions are based on the current available information and are regularly revised as new information accumulates. Countries may need to adapt case definitions depending on their local epidemiological situation and other factors. The interim guideline of WHO published on 20 March 2020 divided COVID-19 disease in suspect case, probable case, and confirmed case. ${ }^{20}$

\section{Clinical syndromes associated with COVID- 19 in Children:}

International pediatric data suggest lower rates of severe coronavirus disease 2019 (COVID-19) in children and higher rates of asymptomatic infection. ${ }^{21}$ Additionally Guan and colleagues explained in their study that mild symptoms or absence of severe symptoms in children may lead to misdiagnosis and will lead to skip the required test for SARS-CoV-2 and therefore, asymptomatic children might spread the disease. It also has revealed that SARS-CoV-2 can be found in feces long after throat and nose swabs test negative. However, the maximum number of SARS-CoV-2 infected children, have been found as a part of family cluster outbreak. ${ }^{22}$ But risk factors for severe disease in pediatric populations have not been clearly identified.

\section{Presentation-}

The typical incubation period of coronavirus disease 2019 (COVID-19) ranges from 1 to 14 days, with an average of 3-7 days (mean, 6.4 days. ${ }^{23}$ According to WHO interim guidance (updated on 27 May, 2020), the following are the clinical symptoms of Covid-19 in children: ${ }^{24}$

- Mild disease (symptoms without evidence of viral pneumonia or hypoxia)

- Moderate disease-Pneumonia

- Severe disease -Severe pneumonia

- Critical disease

- Acute respiratory distress syndrome (ARDS)

- Sepsis

- Septic shock
$\mathrm{Wu}$ and colleagues reported on the clinical characteristics of 68 paediatric patients with COVID-19 in China. ${ }^{25}$ They found that the most common initial symptoms among the 44 symptomatic patients were cough $(32.43 \%)$ and fever $(27.03 \%)$. This finding also highlights the significant number of children with SARS-CoV-2 infection who are asymptomatic. Kidney dysfunction appears to be common among children and adolescents hospitalized with COVID-19 described by Dr. Jelena Stojanovic and colleagues at Great Ormond Street Hospital for Children NHS Foundation Trust, in London. ${ }^{26}$

A study was conducted on children and adolescents ( $\geq 21$ years), hospitalized in children's hospital in New York City, New York, which revealed no association of increased risk of severe disease with younger age (Infancy) and poor immune compromised status of children rather obesity was significantly associated with disease severity. Most patients had fever (80\%), followed by upper/lower respiratory tract symptoms (64\%) and Gastrointestinal tract symptoms (14\%). Most patients were discharged (76\%) with a median length of stay of 3 days (range, 1-30 days). Approximately one-third of the hospitalized patients $(32 \%)$ required some form of respiratory support (eg, nasal cannula, NIPPV, or mechanical ventilation) whereas noninvasive positive-pressure ventilation (NIPPV) was the maximum required respiratory support. ${ }^{27}$ The small sample size of this descriptive study limited the generalized acceptability of the findings.

\section{Skin Manifestation (Covid Rashes):}

A Chinese study published in February reported that skin rashes were present in only 0.2 percent of more than 1,000 COVID-19 patients, while a smaller Italian study published a month later put the prevalence at 20.4 percent. ${ }^{28}$ But diagnosis of COVID rashes in children is currently uncommon compared to adults. In a peer-reviewed paper published in the British Journal of Dermatology, a group of Spanish researchers described five different categories of rashes among COVID-19 patients. $^{29}$

Covid toe-Dermatologists around the world have noticed an increasing number of patients presenting with an unusual rash that may be related to COVID-19: red-purple, tender or itchy 
bumps that develop mostly on the toes, but also on the heels and fingers. ${ }^{30}$

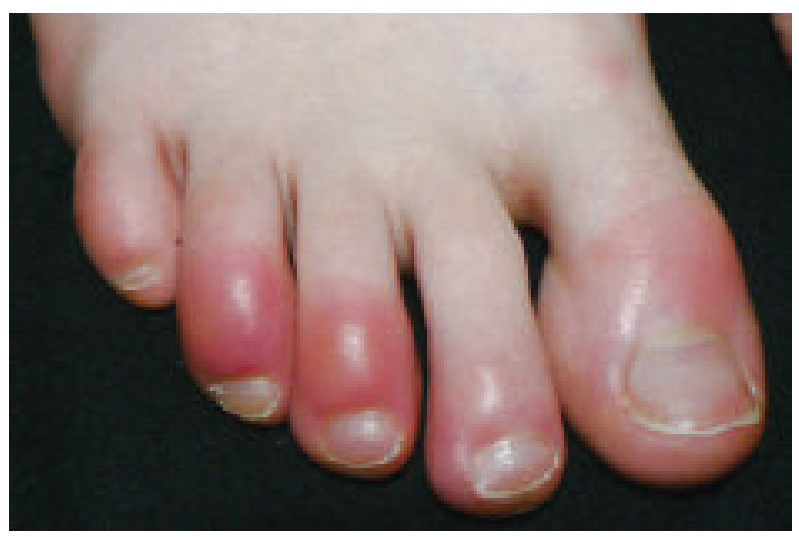

Fig.-2: Covid toe

These rashes, primarily affected younger COVID19 patients and were associated with mild infections. The rash tended to appear later in the illness and lasted an average of 12 days. This is typically associated with exposure to cold conditions. ${ }^{31}$

Other form of reported skin lesions are blisters, urticarial, maculopapular rash, Livedo (also known as necrosis) ${ }^{31}$

In case studies published in the April 30 issue of JAMA Dermatology, doctors identified two additional types of skin conditions that may be associated with COVID-19: petechiae (tiny purple, red or brown spots caused by bleeding under the skin) and a scaly rash called digitate papulosquamous. ${ }^{32,33}$
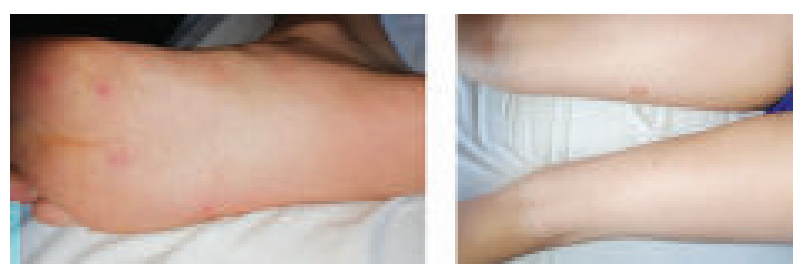

Fig.-3: covid rash

A new, serious COVID-19 cardiovascular presentation emerged in late April and early May 2020 in the form of pediatric multi-system inflammatory syndrome, which includes features similar to Kawasaki disease. It was first reported in the United Kingdom and then cases began to appear in New York City and elsewhere in the United States. On April 26, 2020, clinicians in the U.K recognized increased reports of previously healthy children presenting with a severe inflammatory syndrome with Kawasaki diseaselike features. ${ }^{34}$
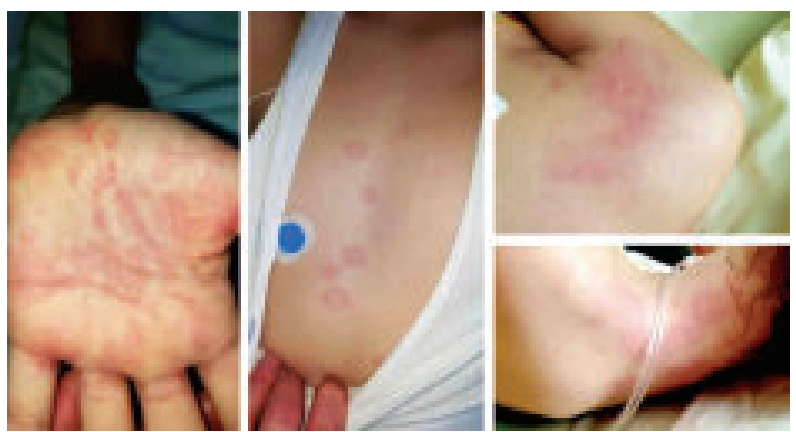

Fig.-4: Rash on the skin of a child who has COVID19 related multi-system inflammatory syndrome (MIS-C). The image is from Damien Bonnet, M.D., Ph.D., Necker Hospital-Université, Paris, who was involved in a Circulation study published on MISC May 14. Image copyright Damien Bonnet, courtesy of the American Heart Association.

\section{Multisystem inflammatory syndrome in children (MIS-C): A burning issue}

\section{Introduction-}

Though children of all ages can become sick with COVID-19, robust evidence associating underlying conditions with severe illness in children is still lacking. Some children who have an active infection with the virus that causes COVID-19 might not show any signs or symptoms at all, but some develop a rare syndrome called multisystem inflammatory syndrome in children (MIS-C). ${ }^{35}$

Onset of MIS-C-

In late April 2020, clinicians in the United Kingdom reported a cluster of eight previously healthy children presenting with cardiovascular shock, fever, and hyperinflammation. ${ }^{36}$ On May 14, 2020, the Centers for Disease Control and Prevention (CDC) issued a national health advisory to report on cases meeting the criteria for multisystem inflammatory syndrome in children (MIS-C). ${ }^{37}$

Epidemiology-36, 38, 39, 40

A prospective and retrospective surveillance were done on paediatric patients with MIS-C admitted to different health centers of US from March 15, 2020, to May 20, 2020 which described organsystem involvement on the basis of symptoms, clinical findings, and laboratory measures. 
The majority of patients (70\%) tested positive for SARS-CoV-2 infection by RT-PCR, antibody testing, or both. Most patients (71\%) had involvement of at least four organ systems and the most commonly involved organ systems were the gastrointestinal (92\%), cardiovascular (80\%), hematologic (76\%), mucocutaneous $(74 \%)$, and respiratory $(70 \%)$ systems. Fever was the commonest symptom among all. Respiratory insufficiency or failure occurred in 59\% cases among $20 \%$ received invasive mechanical ventilation.

Coronary-artery aneurysms were documented in the left anterior descending or right coronary artery in $8 \%$ of the patients. Most patients (92\%) had four or more laboratory biomarkers indicating inflammation. The majority had an elevated erythrocyte sedimentation rate or C-reactive protein level, lymphocytopenia, neutrophilia, elevated ferritin level, hypoalbuminemia, elevated alanine aminotransferase level, anemia, thrombocytopenia and an elevated D-dimer level, prolonged international normalized ratio, or elevated fibrinogen level.

In published case series, many of the pediatric patients with this hyperinflammatory syndrome have had fever and mucocutaneous manifestations similar to those of Kawasaki's disease, a rare vasculitis of childhood that can cause coronaryartery aneurysms.

Until more is known about long-term cardiac sequelae of MIS-C, providers could consider following Kawasaki's disease guidelines for followup, which recommend repeat echocardiographic imaging at 1 to 2 weeks and 4 to 6 weeks after treatment for patients whose disease course is uncomplicated and more frequent echocardiography for patients with coronary-artery aneurysm. Long-term monitoring for other potential sequelae of MIS-C will also be critical.

Some patients have presented with features of toxic shock syndrome, secondary hemophagocytic lymphohistiocytosis, or macrophage activation syndrome.

\section{How is MIS-C treated?}

Most children with MIS-C need to be treated in a hospital, and some will need treatment in a pediatric intensive care unit. They have been treated with anti-inflammatory treatment, including parenteral immunoglobulin and steroids. ${ }^{41}$

Investigations for COVID-19:

WHO recommended following investigations for suspected Covid cases. ${ }^{42}$

A. Detection of virus-

For all suspect cases, collection of upper respiratory tract (URT) specimens (nasopharyngeal and oropharyngeal) for testing by reverse transcription polymerase chain reaction (RT-PCR) and, where clinical suspicion remains and URT specimens are negative, to collect specimens from the lower respiratory tract (LRT) when readily available (expectorated sputum, or endotracheal aspirate/bronchoalveolar lavage in ventilated patient).

B. Radiology and imaging-

- CT Chest- a high-resolution CT is highly preferable. Following are the classical CT findings

o Bilateral involvement in most patients

o Multiple areas of consolidation

o Ground-glass opacities (GGO): bilateral, subpleural, peripheral

o Crazy paving appearance (GGOs and inter-/ intra-lobular septal thickening)

o Bronchovascular thickening in the lesion

o Traction bronchiectasis

- Chest Xray- Is not as sensitive as HRCT. However, this can be done where CT is not available or after doing CT scan if facility is available. Bilateral pneumonia is a common finding of COVID19 pneumonia

- USG of chest- there are specific sonographic findings however it requires a skilled operator who has training on Pulmonary Ultrasonography ${ }^{42}$

C. Supportive investigations:

CBC, CRP and procalcitonin, D-dimer level,blood culture, liver function test and renal function test, ABG analysis, serum ferritin, serum LDH.

\section{Laboratory studies-}

A consistent pattern of laboratory abnormalities has not yet been identified in children with 
confirmed coronavirus disease 2019 (COVID-19). In a study by Wu and colleagues of 68 pediatric patients with COVID-19 in China, 23 children (31.08\%) had abnormal white blood cell counts, and $10(13.51 \%)$ had an abnormal lymphocyte count. Slightly more than half of the children who underwent nucleic acid testing for common respiratory pathogens showed co-infection with pathogens other than SARS-CoV-2. This finding illustrates the need to test for COVID-19 even in the setting of other confirmed viral infections. ${ }^{30}$ Levels of liver and muscle enzymes and myoglobin are increased in some children. Many patients have elevated C-reactive protein (CRP) levels and erythrocyte sedimentation rates (ESR). In severe cases, patients have high D-dimer levels and progressively decreasing lymphocyte counts. ${ }^{27}$

\section{Imaging studies}

Common chest radiograph findings in children with COVID-19 pneumonia include bilaterally distributed peripheral and subpleural ground-glass opacities and consolidation. ${ }^{43}$

A common abnormality seen on computed tomography (CT) of the chest in children with COVID-19 is ground-glass opacity and nodules, which are usually bilateral. ${ }^{44}$

Dr. Marco Denina and colleagues explained that physicians may trust in Lung Ultrasonogram (LUS) findings in the clinical evaluation and follow-up of children with mild to severe COVID-19 as bedside examinations which may reduce both the radiation exposure of the patients and the patients' movement within the hospital, thus lowering the number of healthcare workers and medical devices exposed to SARS-CoV-2. Although additional studies are needed to better understand and characterize LUS findings in this novel disease in children routine LUS can be used as a useful tool in the diagnostic and clinical management of mild or severe COVID-19 in children. ${ }^{45}$

\section{Covid-19 in children: Management Plan}

There is no specific treatment recommended for children by neither WHO, nor the US CDC. However, the aim of the treatment in children with COVID-19 is the prevention of organ failure, ARDS and hospital acquired infections. This is achieved by supportive treatment, which includes adequate intake of fluid, calories, oxygen and ventilator support. ${ }^{46}$
WHO interim guidance is being considered as guideline for clinicians according to case definition. ${ }^{24}$

1. Management of mild COVID-19: symptomatic treatment

\section{Initial management:}

- Managed in primary care/outpatient department, or be encountered during community outreach activities, such as home visits or by telemedicine.

- The decision to monitor a suspect case with mild COVID-19 in a health facility, community facility or home should be made on a case-by-case basis based on the local COVID-19 care pathway.

- If managed at home in self-isolation, counseling is needed according to WHO guidance regarding-personal hygiene, basic Infection Prevention and Control (IPC) measures like maintaining social distance, avoiding direct contact, using face mask, following cough etiquette, daily cleaning and disinfecting surfaces and how to care as safely as possible for the children having COVID19 to prevent the infection from spreading to household contacts.

Symptomatic treatment- Paracetamol, antihistamine, steam inhalation/gurgling of lukewarm water, appropriate rehydration, adequate nutrition, vitamin $\mathrm{C}$ rich diet or supplement, vitamin D (to prevent any respiratory infection) and Zinc (to inhibit intracellular viral replication)

\section{Follow up:}

- Home quarantine for 14 days after clinical recovery.

- Monitor for body temperature and clinical features routinely.

- Caregivers of children with mild COVID-19 should monitor for signs and symptoms of clinical deterioration requiring urgent reevaluation.

2. Management of moderate COVID-19: pneumonia treatment

- Patients with suspected or confirmed moderate COVID-19 (pneumonia) should be isolated to contain virus transmission and they may not require emergency 
interventions or hospitalization; however, isolation is necessary for all suspect or confirmed cases and for patients at high risk for deterioration.

3. Management of severe COVID-19: severe pneumonia treatment

- For All areas where severe patients may be cared for should be equipped with pulse oximeters, functioning oxygen systems and disposable, single-use, oxygendelivering interfaces (nasal cannula, Venturi mask, and mask with reservoir bag).

- Immediate administration of supplemental oxygen therapy is required to any child patient with emergency signs (obstructed or absent breathing, severe respiratory distress, central cyanosis, shock, coma or convulsions) and to any patient without emergency signs and $\mathrm{SpO} 2<90 \%$. The target $\mathrm{SpO} 2$ will be $\geq 94 \%$ during resuscitation and $>90 \%$ once patient is stable. Use of nasal prongs or nasal cannula is preferred in young children, as they may be better tolerated.

- Patients should be monitored closely for signs of clinical deterioration. Haematology and biochemistry laboratory testing and electrocardiogram and chest imaging should be performed at admission and as clinically indicated to monitor for complications, such as acute respiratory distress syndrome and acute liver injury, acute kidney injury, acute cardiac injury, disseminated intravascular coagulation (DIC) and/or shock.

- Patients with COVID-19 should be treated cautiously with intravenous fluids; aggressive fluid resuscitation may worsen oxygenation, especially in settings where there is limited availability of mechanical ventilation.

4. Management of critical COVID-19: acute respiratory distress syndrome (ARDS)

- In selected patients with COVID-19 and mild ARDS, a trial of high-flow nasal oxygen (HFNO), non-invasive ventilation
(NIV) - continuous positive airway pressure (CPAP), bi level positive airway pressure (BiPAP) may be used. If patient acutely deteriorates or does not improve after a short trial (about 1 hour) endotracheal intubation should be performed by a trained and experienced provider using airborne precautions.

5. Management of critical COVID-19: septic shock

- In resuscitation for septic shock in children, $10-20 \mathrm{~mL} / \mathrm{kg}$ crystalloid fluid (normal saline and Ringer's lactate) should be given as a bolus in the first 30-60 minutes. Fluid resuscitation should be curtailed in case of volume overload or in patients with hypoxaemic respiratory failure.

- In children, vasopressors (epinephrine as first line treatment) should be given if signs of fluid overload are apparent or the following persist after two fluid bolus:

o Signs of shock such as altered mental state;

o Bradycardia or tachycardia (HR $<90$ bpm or $>160$ bpm in infants and HR < 70 bpm or $>150$ bpm in children);

o Prolonged capillary refill (> 2 seconds) or feeble pulses;

o Tachypnoea; mottled or cool skin or petechial or purpuric rash; increased lactate; oliguria persists after two repeat boluses;

o Or age-appropriate blood pressure targets are not achieved.

\section{Other supportive measures-}

Doctors may consider following-

- Influenza Vaccine, Zinc, Melatonin, Vit C, Vit D, Oseltamivir.

- Empirical antibiotics when Procalcitonin is high, and neutrophilic leukocytosis

- Treat each syndrome accordingly.

- Use paracetamol as fever-lowering agent.

- Avoid steroids (in mild to severe pneumonia), NSAID 
Note: As there is no high- quality data regarding pharmacological agent, use of these agents should be used judiciously (by judging risk benefit) by consultant while working in COVID 19 hospital.

\section{Care of Covid affected children after discharge: ${ }^{47}$}

\section{Medication after discharge-}

- Treatments for symptoms can be applied if patients have mild cough, poor appetite, thick tongue, coating, etc.

- Those who are on antiviral drugs, can be used after discharge for patients with multiple lung lesions for 3 days after their RT-PCR tested negative

\section{Home isolation-}

Patients must continue two weeks of isolation after discharge.

Dynamic development of newer therapies under research

Therapy for COVID-19 remains experimental in different studies. No drugs or biologics are approved by the US Food and Drug Administration (FDA) for the prevention or treatment of COVID19 in children. Numerous antiviral agents and immunotherapies are being investigated as potential therapies. ${ }^{48}$ Remdesivir gained emergency use authorization (EUA) from the FDA on May 1, 2020, based on preliminary data showing a faster time to recovery of hospitalized patients with severe disease. Safety and effectiveness have not been assessed in children. Pediatric dosing within the EUA was derived based on pharmacokinetic data from adult healthy volunteers. ${ }^{49}$

Philip Zachariah and colleagues administered Remdesivir in hospitalized covid positive children who required escalating respiratory support and it was not associated with any adverse events. However, the small sample size does not allow inference regarding efficacy. The role of immune modulators and steroids remains controversial and should be addressed through clinical trials. ${ }^{27} \mathrm{Bin}$ Chao and colleagues recommended oral lopinavir/ ritonavir along with corticosteroids and aerosolized interferon alpha-2b and also intravenous immunoglobulin, in case of severe cases of HCoVs. ${ }^{50}$
Anti-inflammatory effects of heparin/LMWH may offer benefit and anti-viral mechanisms have been demonstrated for factor Xa inhibitors in animal studies. Consequently, use of empiric therapeutic anticoagulation in certain COVID patients who do not have PE/DVT has been advocated. However, this remains controversial because of the true incidence of $\mathrm{PE} / \mathrm{DVT}$ in patients receiving pharmacological thromboprophylaxis remain uncertain and data to show improved outcomes with therapeutic anticoagulation are lacking. ${ }^{51}$

\section{Prognosis: ${ }^{52}$}

Children mostly have good prognosis, and in mild cases recover 1-2 weeks after disease onset.

Following are the predictors of poor prognosis-

- Age (<1 year), comorbidities (CKD, Malignancy, CHD, DM, Bronchial asthma, NS, CLD etc) are significant predictors of poor clinical outcome

- Lab findings also predict mortality

o High d-dimer

o High ferritin

o High troponin I

o High cardiac myoglobin

o Lymphonia

- Consider following facts:

o Prolonged Mechanical Ventilation

o Complications like : Secondary infection e.g. Ventilator Associated Pneumonia (VAP), Stress Cardiomyopathy (CM), etc

\section{Challenges of Covid-19 for neonates:}

Considerable uncertainty exists regarding the potential for vertical transmission (prenatal/ congenital or perinatal) of SARS-CoV-2 from infected pregnant women to their newborns.

Data from a total of 11 women in China failed to detect virus in maternal whole blood, serum, vaginal mucus, amniotic fluid, and/or breast milk when virus was detectable in maternal nasopharyngeal specimens. ${ }^{53,54}$

Some reports from China found no evidence for mother-to-newborn transmission. ${ }^{53,54}$ In contrast, two additional reports together document detection of SARS-CoV-2 in 4/34 tested newborns. Pneumonia 
was clinically and radiographically diagnosed in these 4 infants and abnormalities in neonatal inflammatory markers and transaminase levels were variably documented. $.55,56$

The interim guidance from the American Academy of Pediatrics supported by guidelines for perinatal care provided by the Centers for Disease Control and Prevention (CDC) and by the American College of Obstetricians and Gynecologists left the following key points regarding perinatal management of neonates exposed to or affected by covid virus. ${ }^{57,58}$

- Neonates can acquire SARS-CoV-2 after birth. Their immature immune system leaves newborns vulnerable to other serious respiratory viral infections, raising concern that SARS-CoV2 may cause severe disease among neonates.

- Airborne, Droplet, and Contact Precautions should be utilized when attending deliveries from women with COVID-19.

- Infants born to mothers with COVID-19 should be tested for SARS-CoV-2 at 24 hours and, if still in the birth facility, at 48 hours after birth. Centers with limited resources for testing may make individual risk/benefit decisions regarding testing.

- A newborn who has a documented SARS-CoV-2 infection (or who remains at risk for postnatal acquisition of COVID-19 due to inability to test the infant) requires admission to an area separated from unaffected infants and if not admitted, frequent outpatient follow-up via telephone, telemedicine, or in-person assessments through 14 days after discharge.

- After hospital discharge, a mother with COVID19 is advised to maintain a distance of at least 6 feet from the newborn, and when in closer proximity use a mask and hand-hygiene for newborn care until (a) she is afebrile for 72 hours without use of antipyretics, and (b) at least 7 days have passed since symptoms first appeared.

- A mother with COVID-19 whose newborn requires ongoing hospital care should maintain separation until (a) she is afebrile for 72 hours without use of antipyretics, and (b) her respiratory symptoms are improved, and (c) negative results are obtained from at least two consecutive SARS-CoV-2 nasopharyngeal swab tests collected e"24 hours apart.

\section{Investigation-}

Other than RT-PCR from nasopharyngeal / oropharyngeal swab the hospital admitted neonates should be thoroughly investigated for other common diseases that may have similar clinical presentations. Investigations might include CBC and Blood culture, CRP, Procalcitonin, CXR, HRCT of chest (in severe cases)

\section{Treatment-}

It includes supportive treatment like other viral diseases in the newborn, treatment of secondary bacterial infection with broad spectrum combination for sepsis as per unit protocol, antiviral drug. Hydroxychloroquine, azythromycin, adjuvant therapies have not been proven to be beneficial in COVID-19 newborn.

Feeding of infants born to mothers with suspected or confirmed SARS-CoV-2: ${ }^{58}$

- Infants born to mothers with suspected, probable or confirmed COVID-19 infection, should be fed according to standard infant feeding guidelines.

- As with all confirmed or suspected COVID-19 cases, symptomatic mothers who are breastfeeding or practicing skin-to-skin contact or kangaroo mother care (KMC) should practice respiratory hygiene and hand hygiene before and after contact with the child, and routinely clean and disinfect surfaces which the symptomatic mother has been in contact with.

- In situations of severe illness, mothers should be encouraged and supported to express milk, and safely provide breast milk to the infant, by uninfected caregivers while applying appropriate Infection Prevention and Control (IPC) measures.

- If parents and caregivers need to be separated from their children, all of them should have access to appropriately trained health or nonhealth workers for mental health and psychosocial support.

Preventive strategies against covid-19: personal level

The best way to help prevent any child from getting COVID-19 virus is to take action to avoid 
exposure to the contact. The following guidelines of the U.S. Centers for Disease Control and Prevention are recommended to prevent onset of Covid-19 or MIS-C in children: ${ }^{41}$

\section{- Keep hands clean}

- Avoid people who are sick (In particular, avoid people who are coughing, sneezing or showing other contagious signs and symptoms).

- $\quad$ Practice social distancing (at least 6 feet/2 meters from other people when outside of your home).

- Wear cloth face masks in public settings

- Clean and disinfect high-touch surfaces every day (This includes doorknobs, light switches, remotes, handles, tables, chairs, desks etc).

- Wash clothing and other items as needed (This includes washable plush toys).

\section{Immunization against Covid $\mathbf{- 1 9 : 5 9}$}

There are tremendous research works going on for bringing a vaccine against Covid-19. Mostly the vaccines were tried on animal models and further studies are required to deliver against SARSCoV-2.

The BCG vaccine has some immune-boosting power and is thought to provide some protection against not just some forms of TB but other diseases as well. Given that health organisations across the world are urgently looking for ways to treat and prevent the spread of COVID-19, until a vaccine is developed, some attention has now turned to the BCG vaccine. However, there is still not enough evidence to draw any conclusions.

Multiple research groups have designed potential vaccines against SARSCoV-2. About 200 groups around the world are working on vaccines and trials are going on. Most experts think a vaccine is likely to become widely available by mid-2021.

Routine childhood vaccination in Covid-19 crisis:

Immunization services are an essential component of health services. Therefore, routine immunization sessions should be maintained as long as COVID-19 response measures allow. Any disruption of immunization services, even for short periods, will result in an accumulation of susceptible individuals: Higher likelihood of VPD (Vaccine preventable disease) outbreak (Especially
Measles \& Polio). Immunization delivery strategies may need to be adapted and should be conducted under safe conditions, without undue harm to health workers, caregivers and the community. 60

Covid-19 Bangladesh perspective:

Onset-

In Bangladesh, first three corona virus cases were identified on $8^{\text {th }}$ March 2020 and first death was recorded on $18^{\text {th }}$ March 2020 who was a 70 years old was with comorbidites.

As of 23 August, total 294,598 covid-19 cases were found in Bangladesh, 179,091 cases recovered and 3,941 cases died. ${ }^{61}$

\section{Hospital based management-}

Different countries have developed their own treatment protocol according to WHO guideline, their own interests and available resources. Bangladesh Government has published its first version of national guideline on $30^{\text {th }}$ May 2020. ${ }^{42}$

According to this guideline cases are of three types, a. Suspected b. Probable c. Confirmed case. For the purpose of treatments patients are usually categorised to Mild, Moderate, Severe and critical illnesses. Mild illness is being treated at home. Moderate, severe and critical illnesses need hospital admission even ICU care.

Currently, there is no available standard treatment protocol, but there are several drugs we are presently using as trials according to institutional guidelines. Other than national guideline, there is Bangladesh Paediatric Association guideline which outlines key principles for the clinical management of children reaching health-care facilities with suspected COVID-19. It is aimed at nationwide general paediatricians treating children during the COVID-19 pandemic.

In Military hospitals Bangladesh "Guideline on COVID-19 by DGMS" is used for management of adult and pediatric population. COVID 19 protocol of children in CMH Dhaka is updated monthly with new additions and sometime deletion of previous concept.

\section{General management:}

From March 2020, COVID-19 has spread worldwide to many Asian and Middle Eastern countries. 
Bangladesh followed international guidelines from the very onset of covid affected patients. Like many other countries we are trying our best by implementing various restrictions like worldwide stopping of International flights. Attention has been focused on quarantining those shown to be infected and asking those potentially infected for selfquarantine. In order to protect the population, the government declared "lockdown" throughout the nation from 23 March to 30 May and prepared some necessary steps to spread awareness to keep this syndrome away from them.

Basic personal hygiene including cough etiquette, the use of disposable tissues and hand hygiene to limit virus spread, with full personal protective equipment necessary(where available) for healthcare staff are being maintained as well. Health regulations for public places like proper using of Masks, maintaining personal hygiene and social distancing should be circulated thoroughly and supervising authority should be there to look after the implementation by the populations and a reward/punishment system must be ensured to raise the awareness among the population.

Routine immunization: Potential actions for Bangladesh

- Following guidelines on COVID 19 infection prevention during immunization sessions

- Prioritizing primary series vaccinations for poliomyelitis containing vaccines, measles rubella and other combination vaccines

- Vaccinating newborns (as per the national immunization schedule) in maternity hospitals

- Prioritizing pneumococcal and seasonal influenza

We the doctors and other health care professionals are the front-line fighters and should play the pivotal role in this critical situation. All the government authorities should act responsibly under the guidance from a single umbrella commanded by a National Committee and of course supervised by a group of Health Care Experts.

\section{Conclusion:}

As the pandemic is new and recent, its clinical manifestations, pathogenesis are still not unveiled fully. Researchers all over the world are working on virus itself, pathogenetic mechanisms, efficacy of drugs, innovation of vaccine, preventive measures etc. It is likely that these viruses will continue to emerge and evolve and cause human outbreaks owing to their ability to recombine, mutate, and infect multiple species and cell types. The disease trajectory in paediatric patients has good prognosis compared to adults as per recent reports. Intensive care unit and deaths are rare. Still additional care may be needed for children with comorbidities and younger children. Bangladesh faces significant challenges in combating COVID-19 as it is a densely populated country. Children are the most vulnerable groups in any crisis situation and therefore continuous monitoring of management and preventive strategies plays a crucial role in this pandemic disaster.

\section{Conflict of Interest - None.}

\section{References:}

1. First Covid-19 case happened in November, China government records show - report. The Guardian. Available from: https://www. theguardian.com/world/ 2020/mar/13/first-covid-19-case-happened-innovember-china-government-records-show-report. Accessed April 2, 2020

2. S. Jiang. A distinct name is needed for the new coronavirus. Lancet 2020; 395 (10228):949.

3. Y. Dong. Epidemiology of COVID-19 among children in China. Pediatrics 2020;145 (6) e20200702; DOI: https://doi.org/10.1542/peds.2020-0702

4. World Health Organization. Coronavirus disease 2019 (COVID-19). Situation Report-74. Updated March 20, 2020. Available from: https:// www.who.int/docs/default-source/ coronaviruse/situation-reports/ 20200321-sitrep-61-covid19.pdf?sfvrsn=6aa18912_2. Accessed July 6, 2020

5. Chinese Center for Disease Control and Prevention. The Epidemiological Characteristics of an Outbreak of 2019 Novel Coronavirus Diseases (COVID-19) - China, 2020 [March 11, 2020]. Available from: http:// www.ourphn.org.au/wp-content/uploads/20200225Article-COVID-19.pdf.

6. Wu Z., McGoogan J.M. Characteristics of and important lessons from the Coronavirus disease 2019 (COVID19) outbreak in China: summary of a report of 72314 cases from the Chinese Center for Disease Control and Prevention. JAMA 2020; 323(13):1239-1242

7. WHO Coronavirus Disease (COVID-19) Dashboard. Available from: https //www.who.int/health-cluster/ news-and-events/news/covid19/en

8. Shrikrushna SU, Ansar QB, Sanap S et al. A REVIEW ON CORONA VIRUS (COVID-19). WJPLS 2020;6(4):109-115. 
9. Harapan H, Itohd N, Yufika A, et al. Review Coronavirus disease 2019 (COVID-19): A literature review. Journal of Infection and Public Health 2020; 13: 667-673.

10. Huang C, Wang Y, Li X, Ren L, Zhao J, Hu Y. Clinical features of patients infected with 2019 novel coronavirus in Wuhan, China. Lancet 2020;395:497506.

11. M. Hoffmann, H. Kleine Weber, S. Schroeder, et al. SARS-CoV-2 cell entry depends on ACE2 and TMPRSS2 and is blocked by a clinically proven protease inhibitor Cell. Immunity 2020;181(2):209-486.

12. Hussin A. Rothan, Siddappa N. Byrareddy. The epidemiology and pathogenesis of coronavirus disease (COVID-19) outbreak. Journal of Autoimmunity 2020; 109: 102433.

13. Ong SW, Tan YK, Chia PY, et al. Air, surface environmental, and personal protective equipment contamination by Severe Acute Respiratory Syndrome Coronavirus 2 (SARS-CoV-2) from a symptomatic patient. JAMA 2020; 323: 1610-1612.

14. Li Y, Zhao R, Zheng S, et al. Lack of Vertical Transmission of Severe Acute Respiratory Syndrome Coronavirus 2, China. Emerging infectious diseases 2020; 26(6): 30360-3

15. Suliman Khan, Liangyu Peng, Rabeea Siddique, Ghulam Nabi, Mengzhou Xue, Jianbo Liu, Guang Han. Impact of COVID-19 infection on pregnancy outcomes and the risk of maternal-to-neonatal intrapartum transmission of COVID-19 during natural birth. Infection Control \& Hospital Epidemiology 2020;41(6):448-450.

16. Lingkong Zeng, Shiwen Xia, Wenhao Yuan, Kai Yan, Feifan Xiao, Jianbo Shao, Wenhao Zhou. Neonatal Early-Onset Infection With SARS-CoV-2 in 33 Neonates Born to Mothers With COVID-19 in Wuhan China. JAMA pediatrics $2020 ; 174(7): 722-725$

17. Bnjamin Lee and William V. Raszka. COVID-19 Transmission and Children: The Child Is Not to Blame. Pediatrics 2020; 146:e20201576

18. Susan R. Weiss, Sonia Navas-Martin. Coronavirus Pathogenesis and the Emerging Pathogen Severe Acute Respiratory Syndrome Coronavirus Microbiology and molecular biology reviews 2005; 69(4): 635-664

19. Y.C. Li, W.Z. Bai, T. Hashikawa. The neuroinvasive potential of SARS-CoV2 may play a role in the respiratory failure of COVID-19 patients. J. Med. Virol 2020: 92 (6): 552-555.

20. Global surveillance for COVID-19 caused by human infection with Coronavirus. Available from: https:// www.who.int/publications/i/item/global-surveillancefor-covid-19-caused-by-human-infection-with-covid-19virus-interim-guidance

21. Lu Xÿp, Zhang Lÿp, Du Hÿp, et al. Chinese Pediatric Novel Coronavirus Study Team. SARS-CoV-2 infection in children. ÿb N Engl J Med 2020; 382(17):1663-1665.

22. Guan WJ, Ni ZY, Hu Y. Clinical characteristics of coronavirus disease 2019 in China. N Engl J Med 2020; 382:1708-1720

23. Shen K, Yang Y, Wang T. Diagnosis, trteatment, and prevention of 2019 novel coronavirus infection in children: experts' consensus statement. World J Pediatr 2020; 16: 223-231

24. Clinical management of COVID-19. Available from: https://www.who.int/publications-detail-redirect/ clinical-management-of-covid-19 Accesed on: May 27, 2020

25. Wu Q, Xing Y, Shi L. Co-infection and other clinical characteristics of COVID-19 in children. Pediatrics 2020; doi: 10.1542/peds.2020-0961

26. Kidney Problems Common in Children With COVID19 - Medscape-Jun 29, 2020. Available from: https:// www.medscape.com/viewarticle/933104

27. Zachariah P, Johnson CL, Halabi KC, et al. Epidemiology, Clinical Features, and Disease Severity in Patients With Coronavirus Disease 2019 (COVID19) in a Children's Hospital in New York City, New York. JAMA Pediatr 2020; doi:10.1001/jamapediatrics. 2020.2430

28. Juan Jimenez-Cauhe, Daniel Ortega-Quijano, Dario de Perosanz-Lobo, et al. Enanthem in Patients With COVID-19 and Skin Rash. JAMA Dermatol 2020; doi:10.1001/jamadermatol.2020.2550

29. Lauren M. Madigan, Robert G. Micheletti, Kanade Shinkai. How Dermatologists Can Learn and Contribute at the Leading Edge of the COVID-19 Global Pandemic. JAMA Dermatol 2020;156(7):733-734.

30. Covid toe and other Covid-19 skin conditions. Available from: https://weillcornell.org/news/covid-toe-and-othercovid-19-skin-conditions Accessed on july 9,2020

31. Several types of skin rashes seen in Covid-19 patients. Available from: https:/www.minnpost.com/secondopinion/2020/05/several-types-of-skin-rashes-seen-incovid-19-patients/Accessed on June 06, 2020

32. Diaz-Guimaraens B, Dominguez-Santas M, SuarezValle A, et al. Petechial skin rash associated with severe acute respiratory syndrome coronavirus 2 infection. JAMA Dermatol 2020; doi:10.1001/ jamadermatol.2020.1741

33. Sanchez A, Sohier P, Benghanem S, et al. Digitate papulosquamous eruption associated with severe acute respiratory syndrome coronavirus 2 infection. JAMA Dermatol 2020; doi:10.1001/jamadermatol.2020.1704

34. Davies P, Evans C, Kanthimathinathan HK, et al. Intensive care admissions of children with paediatric inflammatory multisystem syndrome temporally associated with SARS-CoV-2 (PIMS-TS) in the UK: a multicenter observational study. The Lancet Child and adolescent health 2020; DOI: https://doi.org/10.1016/ S2352-4642(20)30215-7.

35. Multisystem inflammatory syndrome in children (MIS- 
C) and COVID-19. Available from: https:// www.mayoclinic.org/mis-c-in-children-covid-19/art20486809

36. Riphagen S, Gomez X, Gonzalez-Martinez C, Wilkinson $\mathrm{N}$, Theocharis P. Hyperinflammatory shock in children during COVID-19 pandemic. Lancet 2020;395:16071608.

37. Centers for Disease Control and Prevention. Emergency preparedness and response: multisystem inflammatory syndrome in children (MIS-C) associated with coronavirus disease 2019 (COVID-19). Health advisory Available from: https://emergency.cdc.gov/han/ 2020/han00432.asp. opens in new tab.

38. Jones VG, Mills M, Suarez D, et al. COVID-19 and Kawasaki Disease: Novel Virus and Novel Case. Hosp Pediatr 2020. Epub 2020/04/09

39. L.R. Feldstein, E.B. Rose, S.M. Horwitz, et al. Multisystem Inflammatory Syndrome in U.S. Children and Adolescents. N Engl J Med 2020; DOI: 10.1056/ NEJMoa2021680.

40. Qiu H, Wu J, Hong L, Luo Y, Song Q, Chen D. Clinical and epidemiological features of 36 children with coronavirus disease 2019 (COVID-19) in Zhejiang, China: an observational cohort study. Lancet Infect Dis 2020;20:689-696.

41. Centers for Disease Control and Prevention. Emergency preparedness and response: multisystem inflammatory syndrome in children (MIS-C) associated with coronavirus disease 2019 (COVID-19). Health advisory Available from: https://emergency.cdc.gov/han/ 2020/han00432.asp. opens in new tab.

42. National Guidelines on Clinical Management of Coronavirus Disease 2019 (Covid-19) version 4.0, 30 March 2020. Available from: http://www.mohfw.gov.bd/ index.php? option=com _ docman \& task=doc download\&gid=22424\&lang=en Accessed: June 9, 2018

43. Foust AM, Phillips GS, Chu WC. International expert consensus statement on chest imaging in pediatric COVID-19 patient management: imaging findings, imaging study reporting and imaging study recommendations. Radiology: Cardiothoracic Imaging 2020; https://doi.org/10.1148/ryct.2020200214

44. Xia W, Shao J, Guo Y, Peng X, Li Z, Hu D. Clinical and CT features in pediatric patients with COVID-19 infection: Different points from adults. Pediatr Pulmonol 2020;55 (5): 1169-1174.

45. Lung Ultrasound Useful for Tracking COVID-19 in Children - Medscape - Jun 16, 2020. Available from: https://www.medscape.com/viewarticle/932412

46. Zhi-Min Chen, Fu. Jun-Fen, Qiang Shu, et al. Diagnosis and treatment recommendations for pediatric respiratory infection caused by the 2019 novel coronavirus. World J. Pediatrics 2020, 30(6): 201-207

47. Criteria for releasing COVID-19 patients from isolation. Available from: https://www.who.int/news-room/ commentaries/detail/criteria-for-releasing-covid-19patients-from-isolation Accessed: June 17, 2020
48. Sanders JM, Monogue ML, Jodlowski TZ, Curtell JB. Pharmacologic Treatments for Coronavirus Disease 2019 (COVID-19): A review. JAMA 2020; 12: 202-11.

49. FDA. Fact Sheet for Health Care Providers Emergency Use Authorization (EUA) of Remdesivir (GS-4734). FDA.gov. Available from: https://www.fda.gov/media/ 137566/download 2020 May 01. Accessed: July 4, 2020

50. Bin C, Yeming W, Danning W, et al. A trial of lopinavirritonavir in adults hospitalized with severe Covid-19. New Engl. J. Med 2020; 382:1787-1799.

51. Barnes GD, Burnett A, Allen A, et al. Thromboembolism and anticoagulant therapy during the COVID-19 pandemic: interim clinical guidance from the anticoagulation forum.Journal of Thrombosis and Thrombolysis 2020; 50:72-81.

52. Most children with coronavirus have mild symptoms, recover within 2 weeks: study. Available from: https:// www.foxnews.com/health/children-coronavirussymptoms-recover-2-weeks-study. Accessed: July 17, 2020

53. Wang X, Zhou Z, Zhang J, Zhu F, et al. A case of 2019 Novel Coronavirus in a pregnant woman with preterm delivery. Clin Infect Dis 2020. doi.org/10.1093/cid/ ciaa200.

54. Wang S, Guo L, Chen L, et al. A case report of neonatal COVID-19 infection in China [published online ahead of print, 2020 Mar 12]. Clin Infect Dis. 2020;ciaa225. doi:10.1093/cid/ciaa225

55. Dong Y, Mo X, Hu Y, et al. Epidemiological characteristics of 2143 pediatric patients with 2019 coronavirus disease in China. Pediatrics 2020; doi: 10.1542/peds.2020-0702.

56. Zeng L, Xia S, Yuan W, et al. Neonatal Early-Onset Infection With SARS-CoV-2 in 33 Neonates Born to Mothers With COVID-19 in Wuhan, China. JAMA Pediatr. Published online March 26, 2020. doi:10.1001/ jamapediatrics.2020.0878

57. AAP issues guidance on managing infants born to mothers with COVID-19. Available from: https:// www.the-hospitalist.org/hospitalist/article/220319/ coronavirus-updates/aap-issues-guidance-managinginfants-born-mothers Accessed: July 16, 2020

58. Evaluation and Management Considerations for Neonates At Risk for COVID-19. Available from: https:/ /www.cdc.gov/coronavirus/2019-ncov/hcp/caring-fornewborns.html Accessed: July 16, 2020

59. Zimmermann P, Curtis N. An Overview of the Epidemiology, Clinical Features, Diagnosis, Treatment and Prevention Options in Children.The Pediatric Infectious Disease Journal 2020;39(5):355-368.

60. Routine immunization services during the COVID-19 pandemic. Available from: https://apps.who.int/iris/ handle/10665/331925 Accessed: June 17, 2020

61. Coronavirus in Bangladesh-Information about Covid19. Available from: https://www.worldometers.info/ coronavirus/ 